\title{
Estudo do Efeito da Adição de PP Reciclado nas Propriedades Mecânicas e de Escoamento de Misturas de PP/EPDM
}

\author{
Luciene da S. Santos, Antonio H. Monteiro da F. T. da Silva \\ CENPES/PETROBRAS - Cidade Universitária - Rio de Janeiro/RJ \\ Elen B. A. V. Pacheco, Ana L. N. da Silva \\ Instituto de Macromoléculas Professora Eloisa Mano - IMA/UFRJ
}

\begin{abstract}
Resumo: Misturas de polipropileno, PP, e terpolímero de etileno-propileno-dieno, EPDM, são amplamente utilizadas na indústria automobilística, principalmente em parachoques, cuja destinação, após o seu uso, deverá atender a Política Nacional de Resíduos Sólidos. No presente trabalho, o efeito da incorporação de PP reciclado nas propriedades mecânicas e de escoamento das misturas PP virgem/EPDM foi avaliado através de um planejamento fatorial do tipo $2^{\mathrm{n}}$, com $\mathrm{n}=3$ fatores: teor de PP reciclado, perfil de temperatura de processamento e velocidade de rotação da rosca. As variáveis de resposta analisadas foram: módulo de Young, resistência ao impacto e índice de fluidez (MFI). Os resultados mostraram que o aumento da temperatura de processamento tende a aumentar a rigidez, a resistência ao impacto e a fluidez do produto final. $\mathrm{O}$ aumento da velocidade de mistura mostrou uma influência mais significativa na resistência ao impacto do material; enquanto o aumento do teor de reciclado na mistura PP virgem/EPDM levou ao aumento das propriedades mecânicas, bem como do índice de fluidez do produto.
\end{abstract}

Palavras-chave: Reciclagem, mistura PP/EPDM, caracterização.

\section{Study of the Effect of Recycled PP on the Mechanical and Flow Properties of PP/ERDM Blends}

\begin{abstract}
Polypropylene, PP, and ethylene propylene diene copolymer (EPDM) blends are widely used in automotive industry, mainly in bumpers. Their disposal should comply with the rules of the Solid Residue National Policy. In the present study, the effect of recycled PP addition on the mechanical and flow properties of these blends was evaluated using a $2^{\mathrm{n}}$ factorial design, with $\mathrm{n}=3$ factors: recycled PP content, extrusion temperature profile and screw speed. The parameters analyzed were: Young modulus, impact strength and melt flow index (MFI). The results showed that the increase in temperature tends to increase rigidity, impact strength and the flow of the final product. The increase in blend speed showed a significant influence on the impact strength of the material; whereas the increase in recycled PP content led to an increase of the mechanical properties analyzed, as well as of the flow index of the product.
\end{abstract}

Keywords: Recycling, PP/EPDM blends, characterization.

\section{Introdução}

As misturas à base de polipropileno (PP) e elastômero EPDM apresentam grande aplicação na indústria automobilística, especialmente na fabricação de parachoques, revestimentos internos e painéis. Tal demanda se deve ao fato destas misturas apresentarem um balanço adequado entre rigidez e tenacidade ${ }^{[1-3]}$. Para atender as principais exigências técnicas solicitadas pelas montadoras de automóveis, os compostos de PP devem apresentar melhor resistência térmica, com altos valores de resistência ao impacto e menos insumos importados ${ }^{[4]}$. Em consequência do crescimento significativo de produção de automóveis ${ }^{[4]}$ e o aumento de consumo de resinas plásticas pela indústria automobilística ${ }^{[5]}$, fica evidente o aumento no consumo das misturas de PP/EPDM.

Paralelamente torna-se importante ressaltar que foi aprovada a responsabilidade compartilhada pelo ciclo de vida do produto em 2010. Ou seja, os fabricantes, importadores, distribuidores, comerciantes, consumidores e titulares dos serviços públicos de limpeza devem promover o aproveitamento de resíduos sólidos, direcionando-os para a sua cadeia produtiva, reduzir a geração de resíduos e estimular o desenvolvimento de produtos derivados de materiais reciclados. Com isso a indústria automobilística, como todas as empresas, deverá se adequar às exigências legais presentes na Política Nacional de Resíduos Sólidos (PNRS), Lei $12.305^{[6]}$, sobre a responsabilidade desde a aquisição da matéria prima até o descarte dos componentes dos automóveis, como exemplo os parachoques.

Neste sentido, a reciclagem é essencial para a recuperação de recursos naturais utilizados durante o ciclo de vida do parachoque, artefato plástico de maior massa no automóvel ${ }^{[7,8]}$. Além de atender a PNRS, a

Autor para correspondência: Elen B. A. V. Pacheco, Centro de Tecnologia, Instituto de Macromoléculas Professora Eloisa Mano - IMA, Universidade Federal do Rio de Janeiro - UFRJ, Av. Horácio Macedo, 2030, Bloco J, Cidade Universitária, CEP 21941-598, Rio de Janeiro, RJ, Brasil, e-mail: elen@ima.ufri.br 
reciclagem traz outros benefícios, como geração de empregos e aumento da vida útil dos aterros ${ }^{[9]}$.

A literatura ${ }^{[10]}$ mostra um estudo em que foram avaliadas as propriedades mecânicas do copolímero de polipropileno heterofásico virgem, especificado para a fabricação de parachoques, e do material reciclado, proveniente de parachoques descartados. Os resultados comprovaram que a incorporação do material reciclado fragilizou o PP virgem, reduzindo sua deformação na ruptura, embora o produto com $30 \%$ em massa de reciclado tenha apresentado um comportamento próximo ao do material puro. Com relação à propriedade de tensão na ruptura, esse material com $30 \%$ em massa de reciclado atendeu às exigências dos fabricantes de parachoques ${ }^{[10]}$.

Martins et al. ${ }^{[11]}$ avaliaram misturas puras binárias de PP/EPDM e ternárias de PP/EPDM com polietileno de alta densidade, HDPE, e parachoques pós-consumo. Concluíram que os parachoques eram compostos principalmente por PP e EPDM. Observaram presença, em pequena proporção, de HDPE no artefato.

Estudos de misturas virgens de PP/EPDM são encontrados na literatura científica. Suas principais aplicações e propriedades mecânicas ${ }^{[12-19]}$, térmicas ${ }^{[12,20,21]}$, reológicas $^{[22,23]}$ e morfológicas ${ }^{[12,20,24]}$ foram amplamente discutidas.

Contudo, não foram observados estudos na literatura cientifica pesquisada sobre a incorporação de PP pós-consumo em misturas virgens de PP/EPDM, tendo como fase termoplástica um copolímero heterofásico de PP. Sendo assim, a abordagem do presente estudo envolve a avaliação do efeito da incorporação de um material pós consumo, tal como PP proveniente de resíduos de embalagens plásticas, nas propriedades finais de uma mistura usada na confecção de parachoques, PP/EPDM. Esta avaliação foi baseada na aplicação de um planejamento experimental.

Fatores como quantidade incorporada de PP perfil de temperatura e velocidade de extrusão podem exercer influência significativa nas propriedades finais da mistura $\mathrm{PP}_{\text {virgem }} / \mathrm{EPDM}$, o que faz com que experimentos devam ser realizados para a avaliação do grau de influência desses fatores, além de possíveis efeitos sinergéticos e antagônicos entre eles.

De forma a buscar uma matriz otimizada de ensaios, foram avaliados os efeitos principal e de interação de cada um dos fatores que potencialmente impactam nas propriedades finais das misturas $\mathrm{PP}_{\text {reciclado }} / \mathrm{PP}_{\text {virgem }} / \mathrm{EPDM}$.

\section{Experimental}

\section{Materiais}

Os polímeros utilizados neste estudo foram amostras comerciais de copolímero heterofásico de polipropileno - CP 442XP ( $\left.\mathrm{PP}_{\text {virgem }}\right)$; de terpolímero de etileno-propileno-dieno (EPDM) KELTAN 5508, com razão etileno-propileno igual a 70:30\% em massa e teor de etilideno-norboneno (ENB) igual a 4,6\% em massa; e de polipropileno reciclado $\left(\mathrm{PP}_{\text {reciclado }}\right)$, grade de injeção, provenientes de embalagens, sem as especificações do fornecedor. Os materiais foram cedidos gentilmente pela Braskem Petroquímica, DSM Elastômeros do Brasil e PoliInjet, respectivamente.

\section{Planejamento experimental}

O presente trabalho adotou a análise de Variância (ANOVA) com base em um planejamento fatorial do tipo $2^{\mathrm{n}}$, com $\mathrm{n}=3$ fatores (também chamados variáveis de processo).

As variáveis de resposta adotadas para análise foram:

- Incorporação de $\mathrm{PP}_{\text {reciclado }}$ - a sua adição à mistura de $\mathrm{PP}_{\text {virgem }} / \mathrm{EPDM}$ teve por objetivo verificar o efeito da substituição de parte da resina virgem por um material reciclado sobre as propriedades finais da mistura;

- Perfil de temperatura de processamento - esse parâmetro tem efeito direto sobre o grau de mistura entre as fases, bem como na ocorrência, ou não, de processos de degradação;

- Velocidade de rotação das roscas - o efeito da velocidade de mistura está relacionado ao tempo de residência do material dentro da extrusora, impactando no nível de dispersão entre as fases.

A Tabela 1 apresenta os fatores e os níveis adotados para o processamento dos materiais.

As variáveis de resposta adotadas para análise foram: MÓDULO DE YOUNG ( $\left.\mathrm{Y}_{1}\right)$; ÍNDICE DE FLUIDEZ (MFI) $\left(\mathrm{Y}_{2}\right)$; e RESISTÊNCIA AO IMPACTO $\left(\mathrm{Y}_{3}\right)$.

O número de experimentos foi $2^{\mathrm{n}}=2^{3}=8$ experimentos, combinando-se todos os níveis dos diferentes fatores ${ }^{[25]}$.

Os dados de resposta foram analisados estatisticamente com auxílio do programa Statistica, versão 3.0.

\section{Preparação das misturas}

Antes do processamento, os polímeros foram secos, em estufa a vácuo, durante $12 \mathrm{~h}$ e a $50{ }^{\circ} \mathrm{C}$. As misturas foram processadas em extrusora dupla rosca Brabender, $\mathrm{L} / \mathrm{D}=40$ e diâmetro de rosca $(\phi)=20 \mathrm{~mm}$.

Os corpos de prova (em torno de 07 para cada experimento proposto) usados nos testes mecânicos foram preparados em injetora Battenfeld, modelo Plus 35, com perfil de temperatura de $200{ }^{\circ} \mathrm{C}$. A temperatura do molde foi de $50{ }^{\circ} \mathrm{C}$.

Os ensaios de tração foram realizados em Máquina Universal Instron, modelo 5581, com velocidade de separação das garras de $50 \mathrm{~mm} / \mathrm{min}$, de acordo com a norma ASTM D-638 ${ }^{[26]}$, para corpos de prova do tipo IV. A análise de resistência ao impacto foi realizada em equipamento Resil Impactor, da CEAST, baseado na norma ASTM D-256 ${ }^{[27]}$, com martelo de 2 KJ. O índice

Tabela 1. Fatores e níveis estabelecidos no planejamento $2^{\mathrm{n}}$.

\begin{tabular}{ccc}
\hline Fator & Nível Inferior (-1) & Nível Superior (+1) \\
\hline Perfil de temperatura $\left({ }^{\circ} \mathrm{C}\right)-\mathrm{X}_{1}$ & $185 / 200 / 220 / 240 / 260 / 270{ }^{\circ} \mathrm{C}$ (Alto) & $185 / 200 / 220 / 230 / 240 / 250{ }^{\circ} \mathrm{C}$ (baixo) \\
Velocidade de mistura $(\mathrm{RPM})-\mathrm{X}_{2}$ & 40 & 80 \\
Teor de $\mathrm{PP}_{\text {reciclado }}(\% \mathrm{~m})-\mathrm{X}_{3}$ & 30 & 50 \\
\hline
\end{tabular}


de fluidez (03 replicatas para cada ensaio) foi avaliado em equipamento Melt Flow Tester 2000, da CEAST, de acordo com a norma ASTM D-1238 $8^{[28]}$, com carga de $2,16 \mathrm{~kg}$ e temperatura de $230^{\circ} \mathrm{C}$.

\section{Resultados e Discussão}

Os resultados obtidos com os experimentos realizados combinando-se os diferentes níveis dos fatores são apresentados na Tabela 2.

\section{Análise estatística dos resultados}

\section{Análise de Normalidade e homoscedasticidade}

Para a avaliação de Normalidade dos Resíduos, foram realizados os testes de Shapiro-Wilks e Lilliefors, ambos a $\alpha=0,05$. A partir dos resultados obtidos, verifica-se que, segundo o Teste de Shapiro-Wilks, somente para o fator IMPACTO obteve-se $\mathrm{p}=0,0030<0,05$. Para os demais fatores, $p>0,05$ (MFI: $p=0,25$ e Módulo de Young: $\mathrm{p}=0,35$ ), sinalizando a normalidade dos resíduos. Segundo o Teste de Lilliefors, para os 3 fatores obteve-se p $>0,05$ (Impacto: $p<0,1$; MFI: $p<1$ e Módulo de Young: $\mathrm{p}<1$ ), indicativo de normalidade dos resíduos.

A Tabela 3 mostra a avaliação de homogeneidade de variâncias através dos testes de Cochran-Bartlett e Levene's para $\alpha=0,05$.

A avaliação da homogeneidade das variâncias é realizada a partir dos valores de distribuição p. Segundo os testes de Cochran-Bartlet, os dados para as variáveis MÓDULO DE YOUNG e MFI apresentam variâncias homogêneas $(p>0,05)$ e para o teste de Levene's, a propriedade de resistência ao impacto foi a que apresentou dados com variâncias homogêneas. Sendo assim, de um modo geral, pode-se considerar que todos os dados apresentados neste estudo possuem variâncias homogêneas.

Análise de Variância (ANOVA)

Os resultados da análise de variância para variável IMPACTO são apresentados na Tabela 4. Neste caso, todos os efeitos principais foram considerados significativos $(\mathrm{p}<0,05)$, mostrando a importância da análise de todas as variáveis ponderadas neste estudo. É possível também observar a significância dos efeitos de interação entre Perfil de Temperatura e Teor de Reciclado e entre Velocidade e Teor de Reciclado, sendo a primeira interação com efeito suavemente superior. Tais resultados mostram que as variáveis em questão não podem ser analisadas individualmente, visto que o valor do nível em que se encontra um dos fatores irá impactar na influência que o outro fator exerce na resistência ao impacto do produto final.

Os resultados da Tabela 4 mostram ainda que a variação de $185 / 200 / 220 / 240 / 260 / 270{ }^{\circ} \mathrm{C}$ (alto) para $185 / 200 / 220 / 230 / 240 / 250{ }^{\circ} \mathrm{C}$ (baixo) no perfil de temperatura tende a diminuir cerca de $7 \mathrm{KJ} / \mathrm{m}^{2}$ a resistência ao impacto do produto final. $\mathrm{O}$ aumento da velocidade de 40 para $80 \mathrm{rpm}$ e do teor de reciclado incorporado à mistura levaram, individualmente, ao aumento da propriedade em torno de $5 \mathrm{KJ} / \mathrm{m}^{2}$. Avaliando cada fator, dentro da faixa de variação analisada, pode-se concluir que o aumento do perfil de temperatura, bem como o aumento do teor de $\mathrm{PP}_{\text {reciclado }}$ e da velocidade de mistura, provavelmente levou a uma melhora na interação entre as fases $\mathrm{PP}_{\text {virgem }}$ e EPDM, resultando em um aumento da propriedade de impacto do produto final.

O mesmo tipo de análise foi realizado para as variáveis de resposta MÓDULO DE YOUNG e MFI. Os resultados são mostrados nas Tabelas 5 e 6 , respectivamente.

A avaliação da Tabela 5 mostra que para a propriedade MÓDULO DE YOUNG, todas as variáveis de processo estabelecidas foram significativas ( $p<0,05)$, dentro dos valores de níveis analisados, porém, os fatores Perfil de Temperatura e Teor de Reciclado foram os que mais influenciaram na propriedade em questão (maior efeito). Além disso, o efeito de interação entre Perfil de Temperatura e Teor de Reciclado também foi significativo. A variação do perfil 185/200/220/240/260/270 ${ }^{\circ} \mathrm{C}$ (alto) para o perfil 185/200/220/230/240/250 ${ }^{\circ} \mathrm{C}$ (baixo) na extrusão levou a um decréscimo do módulo de $175 \mathrm{MPa}$, enquanto o aumento do teor de reciclado adicionado

Tabela 2. Resultados do Planejamento Fatorial $2^{3}-\mathrm{PP}_{\text {reciclado }} / \mathrm{PP}$ virgen $/ \mathrm{EPDM}$.

\begin{tabular}{ccccccc}
\hline Experimento & $\mathbf{X}_{\mathbf{1}}$ & $\mathbf{X}_{2}$ & $\mathbf{X}_{\mathbf{3}}$ & $\mathbf{Y}_{\mathbf{1}}(\mathbf{M P a})$ & $\mathbf{Y}_{\mathbf{2}}(\mathbf{g} / \mathbf{1 0} \mathbf{m i n})$ & $\mathbf{Y}_{\mathbf{3}}\left(\mathbf{K J} / \mathbf{m}^{2}\right)$ \\
\hline 1 & + & + & + & $871 \pm 62$ & $3,25 \pm 0,01$ & $57 \pm 6$ \\
2 & - & + & + & $676 \pm 60$ & $3,11 \pm 0,01$ & $56 \pm 3$ \\
3 & + & - & + & $890 \pm 26$ & $3,35 \pm 0,01$ & $49 \pm 5$ \\
4 & - & - & + & $518 \pm 34$ & $3,00 \pm 0,01$ & $46 \pm 3$ \\
5 & + & + & - & $717 \pm 22$ & $2,66 \pm 0,00$ & $53 \pm 3$ \\
6 & - & + & - & $640 \pm 27$ & $2,41 \pm 0,01$ & $41 \pm 4$ \\
7 & + & - & - & $706 \pm 10$ & $2,44 \pm 0,00$ & $51 \pm 2$ \\
8 & - & - & - & $612 \pm 14$ & $2,44 \pm 0,00$ & $41 \pm 3$ \\
\hline
\end{tabular}

Y1 = módulo de Young; Y2 = índice de fluidez e Y3 = resistência ao impacto.

Tabela 3. Análise de homogeneidade das variâncias por testes de Cochran-Bartlet e Levene's das variáveis de resposta analisadas $(\alpha=0,05)$.

\begin{tabular}{lccccccc}
\hline Variável de resposta & \multicolumn{3}{c}{ Cochran-Bartlett } & \multicolumn{3}{c}{ Levene's } \\
\hline & Cochran & Bartlett & p & Efeito MS & Erro MS & F & p \\
\hline IMPACTO & 0,297930 & 17,77926 & 0,013 & 25,45645 & 14,53295 & 1,751637 & 0,116 \\
MÓDULO DE YOUNG & 0,243765 & 13,30153 & 0,065 & 3189,891 & 1716,662 & 1,858193 & 0,010 \\
MFI & 0,448537 & 12,71124 & 0,079 & 0,005731 & 0,001259 & 4,551050 & 0,010 \\
\hline
\end{tabular}


Tabela 4. Avaliação dos efeitos sobre a propriedade de IMPACTO $-\mathrm{R}^{2}=0,51$.

\begin{tabular}{cccc}
\hline Fator & Efeito & Erro padrão & P \\
\hline Interseção & $\mathbf{4 9 , 1 1 7 8 9}$ & $\mathbf{0 , 8 0 8 5 7 9}$ & $\mathbf{0 , 0 0 0 0 0 0}$ \\
(1) Perfil Temperatura & $\mathbf{- 6 , 7 8 3 9 1}$ & $\mathbf{1 , 6 1 7 1 5 8}$ & $\mathbf{0 , 0 0 0 0 9 8}$ \\
(2) Velocidade & $\mathbf{4 , 9 6 0 1 5}$ & $\mathbf{1 , 6 1 7 1 5 8}$ & $\mathbf{0 , 0 0 3 3 2 5}$ \\
(3) Teor de Reciclado & $\mathbf{5 , 4 3 1 5 6}$ & $\mathbf{1 , 6 1 7 1 5 8}$ & $\mathbf{0 , 0 0 1 4 1 4}$ \\
(1) e (2) & 0,18611 & 1,617158 & 0,908788 \\
(1) e (3) & $\mathbf{4 , 5 6 3 8 1}$ & $\mathbf{1 , 6 1 7 1 5 8}$ & $\mathbf{0 , 0 0 6 5 9 1}$ \\
(2) e (3) & $\mathbf{4 , 0 8 9 2 4}$ & $\mathbf{1 , 6 1 7 1 5 8}$ & $\mathbf{0 , 0 1 4 2 9 5}$ \\
(1), (2) e (3) & 1,07213 & 1,617158 & 0,510069 \\
\hline
\end{tabular}

Tabela 5. Avaliação dos efeitos sobre a propriedade de MÓDULO DE YOUNG - $\mathrm{R}^{2}=0,63$.

\begin{tabular}{crcc}
\hline Fator & Efeito & Erro padrão & $\mathbf{p}$ \\
\hline Interseção & $\mathbf{7 1 9 , 8 0 0}$ & $\mathbf{1 2 , 7 9 8 9 9}$ & $\mathbf{0 , 0 0 0 0 0 0}$ \\
(1) Perfil Temperatura & $\mathbf{- 1 7 5 , 3 8 9}$ & $\mathbf{2 5 , 5 9 7 9 8}$ & $\mathbf{0 , 0 0 0 0 0 0}$ \\
(2) Velocidade & $\mathbf{5 6 , 2 8 9}$ & $\mathbf{2 5 , 5 9 7 9 8}$ & $\mathbf{0 , 0 3 2 8 3 7}$ \\
(3) Teor de Reciclado & $\mathbf{1 0 1 , 6 4 7}$ & $\mathbf{2 5 , 5 9 7 9 8}$ & $\mathbf{0 , 0 0 0 2 4 4}$ \\
(1) e (2) & 37,295 & 25,59798 & 0,151784 \\
(1) e (3) & $\mathbf{8 9 , 7 3 8}$ & $\mathbf{2 5 , 5 9 7 9 8}$ & $\mathbf{0 , 0 0 1 0 1 3}$ \\
(2) e (3) & 37,079 & 25,59798 & 0,154112 \\
\hline
\end{tabular}

Tabela 6. Avaliação dos efeitos sobre a propriedade de MFI - $\mathrm{R}^{2}=0,94$.

\begin{tabular}{cccc}
\hline Fator & Efeito & Erro padrão & $\mathbf{p}$ \\
\hline Interseção & $\mathbf{2 , 8 3 4 5 8 3}$ & $\mathbf{0 , 0 1 8 5 5 0}$ & $\mathbf{0 , 0 0 0 0 0 0}$ \\
(1) Perfil Temperatura & $\mathbf{- 0 , 1 8 4 1 6 7}$ & $\mathbf{0 , 0 3 7 1 0 0}$ & $\mathbf{0 , 0 0 0 1 4 1}$ \\
(2) Velocidade & 0,050833 & 0,037100 & 0,189551 \\
(3) Teor de Reciclado & $\mathbf{0 , 6 9 0 8 3 3}$ & $\mathbf{0 , 0 3 7 1 0 0}$ & $\mathbf{0 , 0 0 0 0 0 0}$ \\
(1) e (2) & $-0,009167$ & 0,037100 & 0,807987 \\
(1) e (3) & $-0,059167$ & 0,037100 & 0,130317 \\
(2) e (3) & $-0,044167$ & 0,037100 & 0,251220 \\
\hline
\end{tabular}

à mistura favoreceu o aumento de aproximadamente $102 \mathrm{MPa}$ da propriedade em questão. Mais uma vez pode ser evidenciado que o aumento do teor do $\mathrm{PP}_{\text {reciclado }}$ e do perfil de temperatura levou a uma melhora da interação entre os domínios $\mathrm{PP}_{\text {reciclado }}$ e EPDM, resultando também em um aumento da rigidez do produto final.

A análise de significância realizada a partir dos dados apresentados na Tabela 6 mostra que no caso da variável de resposta MFI, os fatores significativos $(\mathrm{p}<0,05)$ foram Perfil de Temperatura e Teor de Reciclado.

A Tabela 6 mostra ainda que a variável Teor de Reciclado foi a que apresentou uma maior influência sobre a propriedade de fluidez do material: o aumento do teor de reciclado levou a um aumento do MFI de cerca de $0,7 \mathrm{~g} / 10 \mathrm{~min}$, ou seja, a incorporação de material reciclado (menor massa molar) à mistura aumentou a fluidez do produto final. Quanto ao efeito do perfil de temperatura: a variação do perfil $185 / 200 / 220 / 240 / 260 / 270{ }^{\circ} \mathrm{C}$ (alto) para o perfil $185 / 200 / 220 / 230 / 240 / 250{ }^{\circ} \mathrm{C}$ (baixo) na extrusão levou a um pequeno decréscimo do valor do MFI (cerca de 0,2 g/10 min). Essa pequena variação pode ser considerada pouco significativa.

\section{Gráficos 2D}

Outra ferramenta importante que pode ser utilizada na avaliação dos efeitos de resposta é a construção de gráficos bidimensionais, relacionando os fatores - Perfil de Temperatura, Velocidade de Rotação e Teor de Reciclado, com as variáveis dependentes - IMPACTO, MÓDULO DE YOUNG e MFI. Esses dados podem ser usados para complementar os resultados obtidos a partir da Análise de Variância (Tabelas ANOVA).

A Figura 1 mostra que o a variação do perfil $185 / 200 / 220 / 240 / 260 / 270{ }^{\circ} \mathrm{C}$ (alto) para o perfil $185 / 200 / 220 / 230 / 240 / 250{ }^{\circ} \mathrm{C}$ (baixo) na extrusão tende a diminuir a resistência ao impacto do produto final e que este comportamento é mais acentuado com a incorporação de menor teor de material reciclado. $\mathrm{O}$ aumento da velocidade de processamento levou a um aumento da resistência ao impacto do produto, especialmente com a adição de um maior teor de $\mathrm{PP}_{\text {reciclado }}$. Em resumo, o uso de um perfil mais alto de temperatura, maior velocidade de processamento e a incorporação de um teor mais elevado de $\mathrm{PP}_{\text {reciclado }}$ levaram a um aumento da propriedade de resistência ao impacto do produto final, nos níveis avaliados. Tal comportamento provavelmente deve-se ao efeito de interação entre as fases $\mathrm{PP}_{\text {virgem }} \mathrm{e}$ EPDM, favorecido pela difusão das cadeias poliméricas (cadeias de tamanhos menores) do $\mathrm{PP}_{\text {reciclado }}$ na massa fundida. Esse efeito permite uma melhor interação entre os domínios de EPDM e PP ${ }_{\text {virgem }}$. 


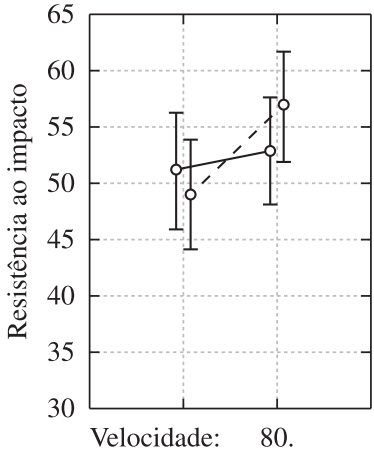

40.

Perfil de temperatura alto

호- Teor de reciclado - Baixo

-호 Teor de reciclado - Alto

Figura 1. Análise Velocidade $\times$ Perfil de Temperatura $\times$ Teor de Reciclado para a variável IMPACTO.

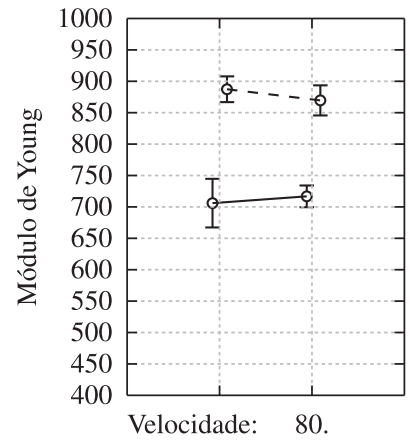

40.

Perfil de temperatura alto

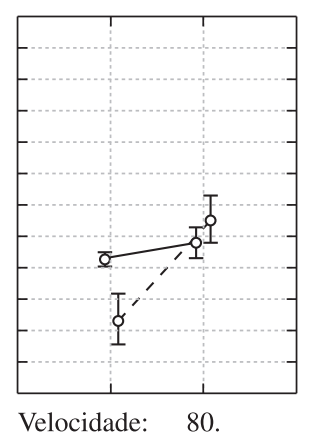

40.

Perfil de temperatura baixo
호- Teor de reciclado - Baixo

-호 Teor de reciclado - Alto

Figura 2. Análise Velocidade $\times$ Perfil de Temperatura $\times$ Teor de Reciclado para a variável MÓDULO DE YOUNG.

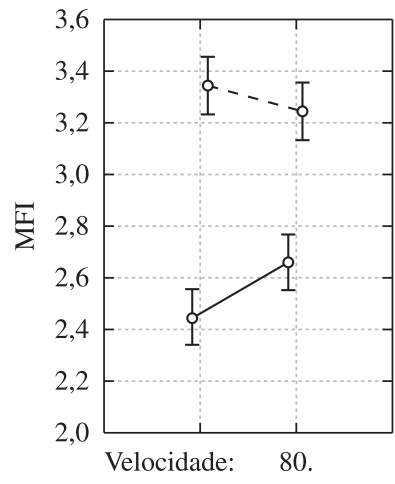

40.

Perfil de temperatura alto

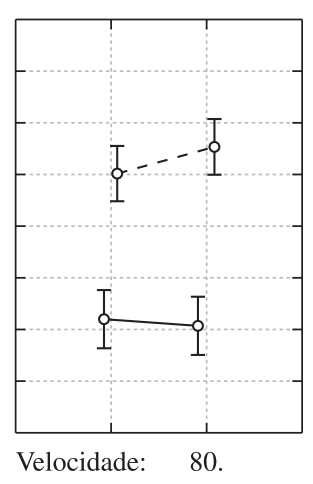

40.

Perfil de temperatura baixo
조- Teor de reciclado - Baixo

-호 Teor de reciclado - Alto

Figura 3. Análise Velocidade $\times$ Perfil de Temperatura $\times$ Teor de Reciclado para a variável MFI.
A literatura ${ }^{[29,30]}$ informa que existe uma exigência, dada pelos fabricantes de automóveis, de um valor mínimo de resistência ao impacto para a aplicação em parachoques. O valor mínimo aceitável para tal aplicação é de $35 \mathrm{~kJ} / \mathrm{m}^{2}$. Nos dois perfis analisados, todas as composições apresentaram valores de resistência ao impacto superiores ao limite mínimo exigido ${ }^{[10]}$. Porém, resultados mais altos foram alcançados em perfil de temperatura e velocidade de mistura mais elevados.

A Figura 2 mostra que para o nível $185 / 200 / 220 / 240 / 260 / 270{ }^{\circ} \mathrm{C}$ (alto) na extrusão, os materiais que foram processados com um maior teor de $\mathrm{PP}_{\text {reciclado }}$ apresentaram maior rigidez (valores mais elevados de módulo de Young), em relação ao produto com menor teor de reciclado. Tendo em vista o erro de análise, pode-se observar que a influência da velocidade não foi tão notável. Observa-se também que a influência da velocidade de rosca se deu de forma mais acentuada para o material com maior teor de $\mathrm{PP}_{\text {reciclado }}$ no perfil de temperatura 185/200/220/230/240/250 ${ }^{\circ} \mathrm{C}$ (baixo) A utilização de uma velocidade menor levou à obtenção de um produto de rigidez, em geral, mais baixa, quando comparado ao material processado nas mesmas condições com maior velocidade. Pode-se dizer que, de um modo geral, nas condições experimentais realizadas, uma mistura $\mathrm{PP}_{\text {reciclado }} / \mathrm{PP}_{\text {virgem }} / \mathrm{EPDM}$ com uma propriedade de maior rigidez pode ser produzida com a incorporação de um maior teor de $\mathrm{PP}_{\text {reciclado }}$ e com o uso de um perfil mais alto de temperatura de processamento, em qualquer uma das duas rotações adotadas.

É possível observar que, nos níveis analisados, a incorporação de $\mathrm{PP}_{\text {reciclado }}$ na mistura $\mathrm{PP}_{\text {virgem }}$ e EPDM levou a um aumento tanto da rigidez como da resistência ao impacto do produto final, sinalizando uma melhora na interação entre as fases PP $_{\text {virgem }}$ e EPDM com a incorporação de $\mathrm{PP}_{\text {reciclado }}$ Tal comportamento evidencia a possibilidade desta mistura ser usada na confecção de parachoques.

A Figura 3 mostra que para os dois níveis de perfil de temperatura analisados, materiais processados com maior teor de reciclado apresentam maiores valores de índice de fluidez. Este comportamento mostra que a incorporação de material reciclado, nas condições analisadas, confere à mistura $\mathrm{PP}_{\text {virgem }} / \mathrm{EPDM}$ um decréscimo da viscosidade, devido a sua baixa massa molar, podendo, assim, favorecer o processamento destes materiais, sem as perdas da integridade mecânica do produto final.

\section{Conclusões}

É possível a produção de um material voltado para a indústria automobilística, especificamente para a confecção de parachoques com um teor considerável de $\mathrm{PP}_{\text {reciclado }}$ O presente estudo também mostrou que é possível agregar valor técnico ao material reciclado obtido a partir de resíduos urbanos para a produção de materiais com sinergismo de propriedades relacionadas à rigidez e resistência ao impacto. Valor ambiental também pode ser obtido com o uso de $\mathrm{PP}_{\text {reciclado }}$ nas composições dos parachoques confeccionados de PP/EPDM, permitindo o atendimento à Política Nacional de Resíduos Sólidos e uma economia no uso de matéria prima virgem. 
A análise do planejamento experimental mostrou que para o estudo em questão, as variáveis de processo que influenciaram positivamente nas variáveis de resposta estabelecidas neste estudo - módulo de Young, resistência ao impacto e MFI foram o Perfil de Temperatura e o Teor de PP

\section{Agradecimentos}

Os autores agradecem às empresas BRASKEM Petroquímica, DSM Elastômeros do Brasil e PoliInjet, pelo fornecimento dos materiais: polipropileno, EPDM e polipropileno reciclado, respectivamente.

\section{Referências Bibliográficas}

1. Rabello, M. - "Aditivação de Polímeros", in: Modificadores de Impacto, cap.12. Editora Artliber, São Paulo (2000).

2. Payne, M. T. \& Rader, C. P. - "Elastomer Technology Handbook", CRC Press, Yest Virginia (1993).

3. Coran, A. Y. \& Patel, R. P. - "Thermoplastic Elastomers", Hanser Publishers, Munick (2004).

4. Associação Nacional dos Fabricantes de Veículos Automotores - Anfavea. - "Anuário da Indústria Automobilística Brasileira", (2011). Disponível em: $<$ http://www.anfavea.com.br/anuario.html $>$. Acesso em: 01 dez. 2011.

5. Associação Brasileira da Indústria Química - ABIQUIM. - "Anuário da Indústria Química Brasileira", (2010).

6. Brasil. Presidência da República. - "Lei no 12.305, 02 de agosto de 2010, Institui a Política Nacional de Resíduos Sólidos", Diário Oficial da República Federativa do Brasil, Brasília (2010).

7. Hemais, C. A. - Polímeros, 13, p.107 (2003). http://dx.doi. org/10.1590/S0104-14282003000200008

8. Fortes, R. G. - "Identificação e avaliação dos principais aspectos relacionados à reciclagem dos plásticos mais utilizados no setor automobilístico brasileiro e o seu atendimento à regulamentação ambiental", Dissertação, Instituto de Tecnologia para o Desenvolvimento e Instituto de Engenharia do Paraná, Brasil (2008).

9. Mano, E. B.; Pacheco, E. B. A. V. \& Bonelli, C. M. C. - "Meio Ambiente, Poluição e Reciclagem", ed. Blucher, São Paulo (2005).

10. Fernandes, B. L. \& Domingues, A. J. - Polímeros, 17, p.85 (2007). http://dx.doi.org/10.1590/S0104-14282007000200005

11. Martins, A. F.; Suarez, J. C. M. \& Mano, E. B. - J. App. Polym. Sci., 75, p.999 (2000). http://dx.doi. org/10.1002/(SICI)1097-4628(20000222)75:8<999::AIDAPP4>3.0.CO;2-Q

12. Hanamoto, L. S. - "Blendas de PP sindiotático com EPDM - Propriedades Físicas e Mecânicas", Tese de Doutorado, Universidade Estadual de Campinas, Brasil (2005).

13. Van Er Wal, A.; Mulder, J. J.; Oderkerk, J. \& Gaymans, R. J. - Polymer, 39, p.6781 (1998).
14. Choudhary, V.; Varma, H. S. \& Varma, K. - Polymer, 32, p. 2534 (1991). http://dx.doi.org/10.1016/0032-3861(91)90332-D

15. Silva, A. L. N. - "Preparação e avaliação térmica, morfológica, mecânica e reológica das propriedades de misturas à base de polipropileno e poli(etileno/octeno)", Tese de Doutorado, Universidade Federal do Rio de Janeiro, Rio de Janeiro, Brasil (1999).

16. Bassani, A.; Persan, L. A. \& Hage, E. - J. App. Polym. Sci., 82, p.2185 (2001). http://dx.doi.org/10.1002/app.2066

17. Stricker, F.; Thomann, Y. \& Mulhaupt, R. - J. App. Polym. Sci., 68, p.1891 (1998). http://dx.doi.org/10.1002/ (SICI) 1097-4628(19980620)68:12<1891::AIDAPP1>3.0.CO;2-G

18. Hung, L.; Pei, Q.; Li, H.; Cheng, F.; Ma, J.; Jiang, S.; An, L.\& Jiang, W. - Polymer, 44, p.3125 (2003).

19. Wang, Y.; Zhang, Q.; Na, B.; Du, R.; Fu, Q.\& Shen, K. - Polymer, 44, p.4261 (2003). http://dx.doi.org/10.1016/ S0032-3861(03)00366-5

20. Silva, A. L. N. - "Estudo de misturas à base de polipropileno e elastômero EPDM", Dissertação, Universidade Federal do Rio de Janeiro, Brasil (1995).

21. Wilhelm, H. M. \& Felisberti, M. I. - J. App. Polym. Sci., 87, p.516 (2003). http://dx.doi.org/10.1002/app.11447

22. Hernández, M.; Albano, C.; Gonzávez, J. \& Ichazo, M. N. - Polym. Bull., 56, p.285 (2006). http://dx.doi. org/10.1007/s00289-005-0481-3

23. Posard-Fillette, M.; Barrés, C. \& Cassgnau, P. - Polymer, 46, p.10256 (2005)

24. Kalló, A. \& Bodor, G. - Polymer, 51, p.1399 (2010).

25. Barros Neto, B.; Scarminio, I. S. \& Bruns, R. E. "Como Fazer Experimentos - Pesquisa e Desenvolvimento na Ciência e na Indústria”, UNICAMP, São Paulo (2003).

26. American Society for Testing and Materials - ASTM. - "D 638: Standard Test Method for Tensile Properties of Plastics", (2010).

27. American Society for Testing and Materials - ASTM. - "D 256: Standard test methods for determining the Izod pendulum impact resistance of plastics", ASTM (2010).

28. American Society for Testing and Materials - ASTM. - "D 1238: Standard test methods for Melt Flow rates of Thermoplastics by extrusion Plastometer", ASTM (2010).

29. Ciuccio, M. T. P. - "Estudo de tendências e oportunidades no desenvolvimento sustentável para a reciclagem de veículos e seus materiais", Dissertação, Universidade Federal de São Carlos, Brasil (2004).

30. Oliveira, A. J. D. - "Caracterização térmica, mecânica e reológica do polipropileno reciclado", Dissertação, Pontifícia Universidade Católica do Paraná, Brasil (2006).

Enviado: $27 / 09 / 11$ Reenviado: 10/04/12 Aceito: 07/05/12 